\title{
Essais
}

ESSAIS

Revue interdisciplinaire d'Humanités

13 | 2018

Écologie et Humanités

\section{L'Espagne et le Parti Socialiste Ouvrier Espagnol face aux défis environnementaux (1982-1986)}

\section{Vincent Marin}

\section{(2) OpenEdition}

12 Journals

Édition électronique

URL : http://journals.openedition.org/essais/504

DOI : 10.4000 /essais.504

ISSN : 2276-0970

Éditeur

École doctorale Montaigne Humanités

\section{Édition imprimée}

Date de publication : 1 janvier 2018

Pagination : 97-104

ISBN : 979-10-97024-03-1

ISSN : 2417-4211

\section{Référence électronique}

Vincent Marin, «L'Espagne et le Parti Socialiste Ouvrier Espagnol face aux défis environnementaux (1982-1986) », Essais [En ligne], 13 | 2018, mis en ligne le 01 décembre 2019, consulté le 12 décembre 2019. URL : http://journals.openedition.org/essais/504 ; DOI : 10.4000/essais.504 


\section{L'Espagne et le Parti Socialiste Ouvrier Espagnol face aux défis environnementaux (1982-1986)}

\section{Vincent Marin}

À l'arrivée du Parti Socialiste Ouvrier Espagnol (PSOE) au pouvoir en novembre 1982, la question de la protection de l'environnement n'avait fait l'objet d'aucune politique publique structurelle en Espagne. Préoccupée avant tout par les enjeux de la démocratisation et par la crise économique frappant le pays, l'Union du Centre Démocratique (UCD) qui avait gouverné entre 1977 et 1982 n'avait pas été sensible aux problématiques environnementales et ce, malgré la dynamique impulsée dans d'autre pays européens depuis la première conférence des Nations unies sur ce sujet célébrée à Stockholm en juin 1972. Mais le faible intérêt pour ces questions était également observable au sein des partis d'opposition, et en premier lieu du PSOE. Les problématiques environnementales n'avaient été en effet que très peu présentes dans les résolutions politiques des Congrès successifs du Parti organisés pendant la Transition démocratique et n'avaient été introduites que très superficiellement dans le programme électoral socialiste. Le point 13 de la seconde partie du programme traitant de l'environnement ${ }^{1}$ demeurait très concis et les propositions relativement limitées et générales : il était question seulement de favoriser la qualité de l'air et de protéger la nature par le biais d'un "plan de dépollution atmosphérique " et d'une "Loi Basique de l'Environnement permettant d'impulser le contrôle et l'utilisation rationnelle de l'environnement et des ressources ${ }^{2}$ ». L'annonce d'une réforme du Code pénal prévoyait par ailleurs " une protection pénale plus efficace de l'environnement ${ }^{3}$ ", sans en préciser les contours.

1 Voir PSOE, Por el cambio. Programa electoral. Partido Socialista Obrero Español, Madrid, PSOE, 1982, p. 31.

2 Traduction de l'auteur, à partir de : « un plan de descontaminación atmosférica " et " une Ley Básica del Medio Ambiente que dé inicio al control y al uso racional del entorno y de los recursos".

3 Traduction de l’auteur, à partir de : « una protección penal más eficaz del medioambiente ». 
À son arrivée aux responsabilités, le chef du Gouvernement socialiste Felipe González ne créa pas de Ministère ou de secrétariat d'État chargé de l'environnement. Contrairement à nombre de pays européens - et notamment la France, qui avait créé un ministère de l'Environnement dès 1971, sous le Gouvernement de Jacques Chaban-Delmas -, il se contenta de maintenir le sous-secrétariat de Disposition du territoire et de l'Environnement placé sous l'égide du Ministère des Travaux publics existant depuis 1977. Mais au cours de la Législature, le PSOE au pouvoir sembla prendre en compte de façon plus précise les problématiques environnementales. L'influence de la Communauté Économique Européenne - avec laquelle l'Espagne était en cours de négociation en vue d'une adhésion à celle-ci - n'était pas étrangère à ces nouvelles préoccupations : dès les années soixante-dix, la protection des espèces, l'amélioration de la qualité de l'air et de l'eau, la prévention des risques environnementaux ou encore la réduction de la pollution figuraient parmi les objectifs des institutions européennes, désireuses d'engager une politique environnementale à échelle continentale. Pour cela, elles avaient fait adopter une série de directives visant à protéger la nature et la biodiversité - comme celle relative à la protection des oiseaux sauvages de 1979 - et à prévenir des risques environnementaux - telle que la directive 96/82/CE, dite directive Seveso.

Les principes qui régissaient et orientaient la politique environnementale communautaire et qui allaient être consacrés par la suite dans l'article 130.2 de l'Acte Unique Européen signé au Luxembourg le 17 février 1986 par les partenaires européens $s^{4}$, furent intégrés dans la politique mise en œuvre par le Gouvernement central, mais aussi par les gouvernements régionaux socialistes 5 . Dans le cadre d'un arsenal législatif inédit en Espagne en matière de protection de l'environnement, le PSOE impulsa des politiques publiques intégrant les concepts d'atteinte à l'environnement et de pollueur-payeur, d'action préventive et de correction par priorité à la source et engagea une nouvelle politique énergétique.

\section{Une législation pénale environnementale}

Conformément à ses engagements électoraux, le PSOE entreprit en début de Législature une réforme du Code Pénal, qui se traduisit par l'adoption de la Loi Organique 8/1983, du 25 juin. Si cette réforme est souvent associée à une volonté d'assouplissement des peines $^{6}-$ le Ministre de la

4 Ce texte intégra au sein du Traité de Rome le titre VII traitant de l'environnement.

5 Parallèlement aux nouvelles dispositions législatives nationales en la matière, plusieurs initiatives régionales virent en effet le jour, telle que la Loi andalouse 6/1984, du 12 juin 1984, créant l'Agence pour l'Environnement.

6 Ce texte visait à alléger et à réduire les sanctions pénales par le biais de la suppression des peines de suspension du permis de conduire, la transformation de plusieurs délits - comme la 
Justice Fernando Ledesma avait affirmé sa volonté d' " humaniser ${ }^{7}$ " la législation en vigueur en la matière -, elle supposa également la mise en œuvre inédite d'une législation pénale environnementale. Le Ministre s'appuya sur l'article 45.1 de la Constitution espagnole selon lequel «tous [les citoyens] ont le droit de jouir d'un environnement adéquat pour le développement de la personne, et ont le devoir de le conserver ${ }^{8}$ " pour mettre en place une législation très coercitive contre les personnes responsables de dégâts environnementaux. S'appuyant sur le principe pollueur-payeur ${ }^{9}$ et sur le concept juridique de " crime contre l'environnement " - connu également sous le nom de "crime environnemental " ou de "crime écologique " - développés dans le droit anglo-saxon et le droit européen dans les années soixante-dix, le Gouvernement socialiste considéra que la législation pénale constituait un des outils contribuant à la protection de l'environnement. Si pour le législateur, " les préceptes pénaux n'all[aient] pas permettre à eux seuls de faire disparaître toute industrie ou activité nocive pour les personnes ou l'environnement ", il était en revanche " évident que toute politique tendant à introduire de la rigueur dans ce problème requé[rait] l'aide coercitive de la Loi pénale ${ }^{10}$ ». Pour cela, l'article 347 bis de la Loi Organique 8/1983, du 25 juin, de réforme urgente et partielle du Code pénal, établit pour la première fois dans l'histoire législative de l'Espagne un " délit contre l'environnement " sanctionnant d'une peine pouvant aller jusqu'à 6 mois de prison ferme et d'une amende de 50000 à 1000000 de pésètes toute personne responsable directement ou indirectement d'émissions de déchets toxiques.

conduite sans permis - en simples infractions, la dépénalisation de la stérilisation volontaire, l'allègement des peines en cas de délits contre la propriété, ou encore la réforme du délit contre l'honnêteté. Ces mesures d'assouplissement des sanctions et de réduction du nombre de peines s'accompagna par ailleurs d'une individualisation dans la définition des sanctions par le biais de la prise en compte du contexte social de la personne poursuivie, en particulier en matière de trafic de drogue et de stupéfiants.

7 Traduction de l'auteur, à partir de : « Humanizar el Código Penal». Voir s.n., "El ministro de Justicia descarta una futura ampliación de los supuestos actuales de despenalización del aborto ", El País, 3 février 1983.

8 Traduction de l'auteur, à partir de : « Todos tienen derecho a disfrutar de un medio ambiente adecuado para el desarrollo de la persona, así como el deber de conservarlo ».

9 Ce principe avait été adopté en 1972 par l'Organisation de Coopération et de Développement Économiques (OCDE) dont l'Espagne faisait partie et figurerait par la suite dans l'Acte Unique européen.

10 Traduction de l'auteur, à partir de : «Unos preceptos penales no han de poder por sí solos lograr la desaparición de toda industria o actividad nociva para personas o medio ambiente; pero también es evidente que cualquier política tendente a introducir rigurosidad en ese problema requiere el auxilio coercitivo de la Ley penal». Voir préambule de la Loi Organique 8/1983. 


\section{Le principe de correction par priorité à la source}

Alors que rien n'avait été annoncé en ce sens dans le programme électoral, le Gouvernement s'engagea dans une politique visant à supprimer à la source les substances susceptibles d'avoir un impact environnemental sur l'air ou sur l'eau en diminuant l'existence de produits polluants et en privilégiant des ressources et des techniques moins polluantes. Cette politique répondait étroitement aux recommandations du Conseil européen, qui aborda ces questions dans le cadre de sommets européens notamment à Dublin en décembre 1984 et à Bruxelles les 29 et 30 mars 1985 et adopta une série de mesures en ce sens à l'échelle continentale. Une des conclusions tirées par le Conseil était « la nécessité d'une action cohérente des États membres dans le cadre communautaire, dans les domaines de la protection de l'atmosphère, de la mer et du sol ${ }^{11}$ ». Alors que l'adhésion de l'Espagne aux différentes institutions européennes n'était pas encore effective, les instruments et les objectifs fixés par les partenaires européens furent intégrés dans la législation espagnole dès 1985.

Ainsi, la réduction du plomb dans l'essence constitua une mesure adoptée par le Gouvernement socialiste avant même l'intégration effective dans la CEE. Dans une perspective d'harmonisation de la législation espagnole avec celle de ses voisins européens, il adopta le Décret royal 284/1985, du 20 février, qui fixa de nouvelles règles pour les différents types d'essence des véhicules automobiles ${ }^{12}$. Ce texte évoquait explicitement une mise en conformité avec « la pratique habituelle de tous les pays européens de [l'] entourage [de l'Espagne] ${ }^{13}$ " et était étroitement liée à la politique communautaire : sous l'impulsion de la République Fédérale d'Allemagne, le Parlement européen avait adopté le 6 juin 1983 une résolution visant à réduire et, à terme, à faire disparaître la présence de plomb dans l'essence et en décembre 1984, les ministres des pays membres de la CEE avaient accepté la proposition de la Commission européenne visant à obliger les stations-services à disposer d'essence sans plomb à partir du $1^{\text {er }}$ janvier 1989. Ainsi, pour la première fois dans l'histoire de l'Espagne, la raffinerie Petronor mit en vente dès 1986 une essence sans plomb qui avait vocation à réduire la pollution atmosphérique et qui était destinée en priorité aux nouveaux véhicules disposant de catalyseurs.

11 Voir le rapport intitulé Conseil européen des 29-30 mars 1985 à Bruxelles : élargissement-situation économique et sociale - renforcement de la base technologique et de la compétitivité de l'industrie communautaire - environnement - faim dans le monde - l'Europe des citoyens - PIM - questions institutionnelles.

12 Cette législation fut complétée peu de temps après, en début de III Législature, par le Décret royal 2482/1986, du 25 septembre.

13 Traduction de l'auteur, à partir de : " la práctica habitual de todos los países europeos de nuestro entorno ». 
Par ailleurs, l'influence communautaire fut notable lorsque le Gouvernement entreprit de rédiger la Loi 20/1986, portant sur les résidus toxiques et dangereux, qui entra en vigueur le 14 mai 1986. Première loi générale consacrée exclusivement à des préoccupations écologiques votée en Espagne, cette législation fixa le régime juridique de la gestion des résidus toxiques et dangereux pour la protection des ressources naturelles ainsi que les responsabilités, infractions et sanctions en la matière. Réformant la Loi 42/1975 qui abordait la question du ramassage et du traitement des résidus solides urbains, elle s'inspira en grande partie du Droit communautaire : elle intégra en effet les grandes lignes de la Directive 1975/442/CEE, du 15 juillet, relative aux résidus et transposa dans la législation espagnole la Directive 1978/319/CEE, du 20 mars, relative aux résidus toxiques et dangereux.

\section{Vers une nouvelle politique énergétique}

Dans son programme électoral, le PSOE souhaitait « donner un plus grand poids relatif au charbon et au gaz naturel et un poids inférieur à l'énergie nucléaire ». En se limitant à faire référence à des énergies fossiles et à l'énergie nucléaire, le programme socialiste faisait donc exclusivement référence à des sources d'énergies non renouvelables, à l'exception près d'une référence à "l'expansion de nouvelles énergies propres renouvelables ${ }^{14}$ " dans le domaine de la recherche. La création d'un nouveau Plan énergétique national permit de renforcer la prise en compte d'une dimension écologique dans la production d'énergie, sans pour autant faire le choix d'une orientation claire sur la question du nucléaire.

En effet, la politique nucléaire du Gouvernement espagnol ne fut pas dépourvue d'ambiguïté. Dans la lignée de ce que s'était proposé la Suède en prônant en 1980 l'abandon de l'énergie nucléaire et la fermeture de centrales, les socialistes souhaitaient « mener à bien une politique de stricte complémentarité de l'énergie nucléaire, qui [répondrait] exclusivement aux besoins de consommation non couverts par d'autres sources disponibles » et opérer " un arrêt ordonné du programme nucléaire en $\operatorname{cours}^{15}$ ". Conformément aux engagements électoraux, le Gouvernement ordonna un moratoire sur le nucléaire en juin 1984 qui s'inscrivait dans le cadre de la présentation de son Plan

14 Traduction de l'auteur, à partir de : «Desarrollar la tecnología nacional en todo el sector, poniendo el máximo empeño en la expansión de las nuevas energías limpias y renovables, con especial atención a la energía solar".

15 Traduction de l'auteur, à partir de : " dar un mayor peso relativo al carbón y al gas natural y menor a la energía nuclear ", "llevar a cabo una política de estricta complementariedad de la energía nuclear, que atenderá exclusivamente las necesidades del consumo no cubiertas con otras fuentes disponibles " et " una detención ordenada del programa nuclear en curso ». Voir PSOE, Por el cambio. Programa electoral. Partido Socialista Obrero Español, Madrid, PSOE, 1982, p. 12. 
Énergétique National 1983-1992 visant à opérer un ajustement énergétique. Ce plan remplaçait le Plan énergétique de 1978-1987 - dont les prévisions de la demande en énergie avaient été surévaluées - et visait à limiter le poids de l'énergie nucléaire à $7500 \mathrm{MW}$, soit 5000 de moins que le plan précédent. Justifié par l'impossibilité d'éliminer les résidus radioactifs, le moratoire se traduisit par l'arrêt de la construction de cinq des sept projets de centrales nucléaires : les centrales nucléaires de Lemóniz I et II, Valdecaballeros I et II et Trillo $\mathrm{II}^{16}$. Seuls les projets de la centrale de Trillo I et la centrale nucléaire de Vandellós II furent poursuivis. Mais parallèlement à la baisse radicale de l'investissement en matière d'énergie nucléaire, le Gouvernement socialiste négocia les modalités de son adhésion à la Communauté Européenne d'Énergie Atomique (CEEA) ou EURATOM, organisation née de la signature d'un des Traités de Rome le 25 mars 1957 et dont la structure exécutive avait été fusionnée le $1^{\text {er }}$ juillet 1967 avec la CEE et la CECA avec l'adoption du Traité de Bruxelles signé le 8 avril 1965. Cette organisation avait précisément pour vocation de favoriser, à des fins civiles, l'essor de l'industrie nucléaire et de coordonner les programmes de recherche sur l'énergie nucléaire afin de répondre aux besoins énergétiques. Si la question de l'intégration de l'Espagne dans l'EURATOM n'avait pas du tout été abordée dans le programme électoral, elle constituait en revanche un des chapitres de la négociation dans le cadre de l'entrée de l'Espagne dans la CEE. Le 19 décembre 1983, un accord entre les négociateurs espagnols et leurs partenaires communautaires fut conclu. Dans la négociation sur l'EURATOM, les négociateurs espagnols luttèrent d'arrache-pied pour ne pas avoir l'obligation de souscrire au Traité sur la non-prolifération des armes nucléaires ${ }^{17}$ et ce, alors même qu'à la veille des élections, le PSOE s'était engagé à étudier favorablement l'opportunité pour l'Espagne de le ratifier ${ }^{18}$ : bien qu' étant favorable au maintien du principe de non nucléarisation du territoire espagnol, Felipe González fit finalement le choix, pour des raisons politiques, de ne pas y adhérer, au prétexte qu'il ne souhaitait pas faire allégeance aux superpuissances. Finalement, l'adhésion à l'EURATOM, opérée au même moment que l'adhésion à la CEE et à la CECA, supposa pour l'Espagne un changement d'orientation radical en matière d'énergie nucléaire, puisqu'elle s'engagea à respecter la réglementation communautaire en matière de recherche, d'approvisionnement, d'investissement et de sécurité mais bénéficia également de prêts de l'EURATOM destinés au développement de l'énergie nucléaire ${ }^{19}$. Cela eut pour consé-

16 Un processus d'indemnisation des entreprises propriétaires de ces centrales - berdrola, Endesa, Unión Fenosa et la Compagnie Sévillane d'Électricité - allant jusqu'en 2020 s'engagea alors.

17 Ils obtinrent gain de cause grâce au soutien de la France sur ce point.

18 Voir PSOE, Por el cambio. Programa electoral. Partido Socialista Obrero Español, Madrid, PSOE, 1982, p.45.

19 Voir Sanz Díaz, Benito, Centrales nucleares en España: el parón nuclear, Valence, Fernando Torres, 1984. 
quence un changement de discours des socialistes espagnols sur cette énergie : s'alignant sur le positionnement des institutions européennes, ils soutinrent en fin de Législature que le développement de la filière nucléaire ne rentrait pas en contradiction avec les principes de précaution et d'action préventive qu'ils préconisaient par ailleurs.

Parallèlement, le Gouvernement fit le choix d'une politique de diversification de l'offre énergétique, politique qui ne figurait pas dans le programme électoral. Le Plan énergétique défendu par le Ministre de l'Industrie et des énergies Carlos Solchaga intégra en effet les énergies renouvelables au sein de la nouvelle politique énergétique et fixa pour la première fois des objectifs chiffrés pour leur développement. Pour le PSOE, ces énergies présentaient à la fois des avantages sur le plan environnemental et la possibilité de conquérir de nouveaux marchés. Si les socialistes espagnols n'étaient pas pionniers en la matière - l'énergie hydroélectrique, l'énergie de biomasse, l'énergie solaire, la géothermie, les énergies marines et surtout l'énergie éolienne, avaient été développées dès les années 70 à la suite des crises pétrolières aux États-Unis, en Allemagne, en Suède, au Canada ou encore au Royaume-Uni-, ils conduisirent en revanche une politique inédite en Espagne.

En définitive, les initiatives du premier Gouvernement socialiste en matière de protection environnementale s'inscrivirent dans une large mesure dans une dynamique de prise en compte des défis écologiques à l'échelle continentale. La perspective de l'entrée de l'Espagne dans la CEE - qui fut effective le $1^{\text {er }}$ janvier 1986 - et la volonté de développer des politiques de convergence avec les autres nations européennes contribuèrent en effet à faire émerger une sensibilité écologique au sein de l'identité du socialisme espagnol. La volonté de prise en compte des défis écologiques fut perceptible dans les résolutions du XXX Congrès fédéral du PSOE organisé en décembre 1984, révélant ainsi l'assimilation progressive d'une dimension écologiste inédite dans le corps doctrinal du PSOE ${ }^{20}$. Mais s'il manifesta un intérêt croissant pour la protection de l'environnement qui se traduisit par un renforcement programmatique sur ces sujets à l'occasion des élections générales du 22 juin 1986, le positionnement du PSOE sur l'énergie nucléaire refléta néanmoins une certaine forme de suivisme.

20 Cette dimension se vit d'ailleurs confirmée lors des législatures postérieures par des mesures concrètes. Parmi elles, la Loi 2/1988, du 28 juillet, dite de Côtes ou encore la Loi 4/1989, du 27 mars, relative à la conservation des espaces naturels, de la flore et de la faune sauvage. 
En cela, la défense de l'environnement ne constitua pas pour le socialisme espagnol une composante structurelle de son identité politique : loin de théoriser ou de mettre en œuvre un "éco-socialisme " ou un "socialisme vert ", le PSOE au pouvoir jusqu'en mai 1996 continua dans une large mesure, lors des législatures successives, à subordonner la politique environnementale aux directives européennes ${ }^{21}$.

\title{
Vincent Marin \\ INU Jean-François Champollion d'Albi vincent.marin@univ-jfc.fr
}

\section{Résumé}

En Espagne, les enjeux écologiques furent pris en compte assez tardivement par les responsables politiques. Les premières politiques publiques menées en la matière furent celles mises en ouvre par le premier gouvernement socialiste de l'Espagne démocratique, conduit par Felipe González entre 1982 et 1986. L'influence de la Communauté Économique Européenne est déterminante pour comprendre les différentes mesures en faveur de l'environnement impulsées par le Parti Socialiste Ouvrier Espagnol, dont le corps doctrinal et programmatique n'avait jusqu'alors que très peu intégré les problématiques écologiques.

\section{Mots-clés}

Environnement, politique, Espagne, socialisme, PSOE.

\begin{abstract}
Spanish political leaders started to take ecological issues into consideration relatively late. Early governmental policies dealing with ecology were implemented in democratic Spain by the first Socialist party in power, under the leadership of Felipe González, between 1982 and 1986. The influence of the European Economic Community is very important to understand the various environmental measures introduced by the Spanish Socialist Worker's Party who, until that time, had not included ecology in their ideological agenda.
\end{abstract}

\section{Keywords}

Environment, Politics, Spain, Socialism, Spanish Socialist Worker's Party.

21 Par la suite, la majeure partie de la politique environnementale mise en œuvre au cours des deux législatures successives du Parti Populaire de José María Aznar s'inscrivit dans cette même dynamique : la législation en matière d'emballages, de déchets, de prévention et de réduction de la pollution ou encore de forêts adoptée entre 1997 et 2003 répondait à des directives communautaires. 